\section{Aborto, ¿es un problema de salud pública en Chile en el campo de la salud materno-perinatal?}

\author{
MARÍA TERESA VALENZUELA, PAMELA SAN MARTÍN P.a , \\ GABRIEL CAVADA ${ }^{\mathrm{b}}$
}

\section{Is abortion a serious public health problem in Chile in the field of maternal-perinatal health?}

Background: The World Health Organization, by 2014, estimates that approximately 22 million unsafe abortions take place every year in the world, almost all of them in developing countries. The Millennium Goals, as part of the fifth compendium, focused on maternal health by proposing that member states should reduce maternal mortality to $75 \%$ by 2015. Aim: To determine, using maternal health indicators, if abortion in Chile is a priority health problem. $\mathbf{M a}$ terial and Methods: Data about maternal mortality and its causes between 1982 and 2014, was obtained from the databases available at the Chilean Ministry of Health. Trend analyzes were carried out using linear autoregressive models. Results: Between 1982 and 2012, maternal mortality rates decreased from 51.8 to 18.3 per 100,000 live births. Complications of pregnancy, childbirth and puerperium were the first three causes and the last one is abortion. The proportion of abortions due to unspecified causes, including induced abortion, decreased from $36.6 \%$ to 26.1\% between 2001 and 2012. Conclusions: Abortion is not a public health problem in Chile. To continue reducing maternal mortality, programs for the early detection of risks such as diabetes, obesity and hypertension should be implemented.

(Rev Med Chile 2017; 145: 1013-1020)

Key words: Abortion, Induced; Chile; Health Priorities; Maternal Mortality; Public Health.
Departamento de Salud Pública

y Epidemiología, Facultad de

Medicina, Universidad de los

Andes. Santiago, Chile.

Ingeniero estadístico.

bBioestadístico.

Recibido el 24 abril de 2017,

aceptado el 18 agosto de 2017 .

Correspondencia a:

Dra. María Teresa Valenzuela

Facultad de Medicina,

Universidad de los Andes

Av. Mons. Álvaro del Portillo

12.455. Las Condes, Santiago,

Chile.

mtvalenzuela@uandes.cl
L

a promoción del cuidado materno ha sido un pilar fundamental en la elaboración de $\checkmark$ políticas públicas alrededor del mundo, particularmente interpuestas por los "Objetivos del Milenio" como parte del quinto compendio que se enfocó en la salud materna. Las Naciones Unidas plantearon como meta que todos los estados deben disminuir la mortalidad materna en $75 \%$ para 2015, en cambio, esta ha disminuido en $50 \%$ globalmente ${ }^{1}$.

Según cifras de la Organización Mundial de la Salud (OMS), actualizadas al 2014, calculan que cada año se realizan unos 22 millones de abortos inseguros en todo el mundo, casi todos ellos en países en desarrollo. Las defunciones debidas a "abortos peligrosos" representan 13\% de todas las defunciones maternas ${ }^{2}$.

Cada año, unos 5 millones de mujeres ingresan a hospitales como consecuencia de un aborto peligroso y más de tres millones de mujeres que han sufrido complicaciones no reciben atención médica ${ }^{2}$.

Sesenta y un países $(39,5 \%$ de la población mundial) permiten el aborto sin restricción en cuanto a razón, 66 países $(25,5 \%)$ lo permiten para salvar la vida de la mujer o está prohibido 
totalmente, 59 países $(13,8 \%)$ lo permiten para preservar la salud de la mujer, 13 países $(21,3 \%)$ lo permiten por razones socioeconómicas, y sólo cinco estados de los 193 que integran la Organización de las Naciones Unidas (ONU) prohíben totalmente el aborto: Malta, Ciudad del Vaticano, El Salvador, Nicaragua y Chile 3 .

En Chile, actualmente, se está discutiendo un proyecto de ley que regula la despenalización de la interrupción voluntaria del embarazo en tres causales:

1. Frente a riesgo vital de la madre.

2. Por malformaciones fetales incompatibles con la vida.

3. Por embarazos originados por casos de violación sexual.

Chile ha asumido importantes compromisos con organismos internacionales, así como con nuestra propia población, a través de las metas sanitarias. Para el período 2000-2010, la meta fue reducir la tasa de mortalidad materna (TMM) en $50 \%$, es decir reducirla desde $23,0 * 100.000$ nacidos vivos (NV) a $12^{\star} 100.000 \mathrm{NV}^{5,6}$.

Entre los años 2011 y 2020 no hubo un objetivo de la década en torno a este indicador, sin embargo, se ha trabajado por cumplir la meta del "Objetivo del Milenio", de lograr una TMM de $10^{\star} 100.000 \mathrm{NV}$ al $2015^{6}$.

Para responder si es el aborto una causa importante de la mortalidad materna y es, por ende, un problema de salud pública en Chile, se analizaron indicadores de salud y la tendencia de ellos, directamente relacionados con el aborto y otros en forma indirecta.

\section{Material y Método}

Estudio ecológico de series de tiempo cuyo período comprendió los años 1982 y 2012.

Para la obtención de los datos, se usó información desde el Departamento de Estadísticas e Información de Salud (DEIS) de Chile ${ }^{7}$.

Para el análisis se registraron las muertes maternas, las muertes maternas por causas específicas y los egresos hospitalarios de acuerdo a la Clasificación Internacional de Enfermedades (CIE-10) ${ }^{8}$.

La mortalidad materna se expresó como el número de muertes maternas que ocurren durante el embarazo y hasta 42 días después del parto respecto a los recién nacidos vivos (RNV), amplificado por 100.000. Estas muertes maternas ocurren durante el embarazo, parto o puerperio, por causas propias de la gestación o agravadas por esta, excluidas las causas accidentales e incidentales. Para calcular la mortalidad materna por causas específicas se extrajo la información de acuerdo a CIE 10, códigos O00 a O99, graficándose las causas más relevantes y amplificada por $100.000 \mathrm{RNV}$. En la Tabla 1 se muestra la codificación de muertes maternas por causas específicas. Para efectos de egresos hospitalarios, se analizó la información oficial proporcionada por el DEIS de los egresos

Tabla 1. Codificación de muertes maternas y egresos hospitalarios según CIE-10

\begin{tabular}{|ll|}
\hline Código CIE-10 & Causa de muerte materna \\
\hline O00-008 & Aborto \\
\hline 010-016 & Edema, proteinuria y trastornos hipertensivos en el embarazo, parto y el puerperio \\
\hline O20-; 046.; 067.; 072. & Hemorragia en el embarazo, parto y el puerperio \\
\hline $\begin{array}{l}\text { O21-026; 029-045; 047-048; } \\
\text { 060-066; 068-071; 073-075 }\end{array}$ & Complicaciones predominantes, relacionadas con el embarazo y el parto \\
\hline 085.-; 086.-; 091. & Sepsis puerperal y otras infecciones \\
\hline 087-090; 092. & Complicaciones relacionadas con el puerperio \\
\hline 095. & Muertes obstétricas de causa no específica \\
\hline 096.-; 097 & Muerte materna debida a cualquier causa obstétrica que ocurre después de 42 días \\
\hline 098.-; 099.- & pero antes de un año del parto \\
\hline
\end{tabular}


por abortos, dentro de los cuales se encuentran los abortos no especificados, cuyo código es O06 y en lo que eventualmente pudiesen estar incluidos los abortos inducidos.

Por último, se analizaron los datos sobre muertes por anomalías congénitas en el total de muertes infantiles. Entre las anomalías congénitas estarían incluidas causas incompatibles con la vida, más aun las que causan muertes en el período neonatal precoz.

Los análisis de tendencia se realizaron a través del modelo lineal autoregresivo de primer orden (Regresión de Prais-Winsten) ${ }^{9}$, usando el software estadístico Stata versión 13.0, con nivel de significación de $5 \%$.

\section{Resultados}

La tasa de mortalidad materna en Chile ha descendido de 51,8 a 18,3 en el período 1982-2012. Se deduce que el descenso de este indicador ha sido exponencial, $\mathrm{p}=0,0000$, con una calidad del ajuste de $92,6 \%$ (raíz cuadrada de $\mathrm{R}^{2}$ ) (Figura 1 ).

La tasa del año 2011 fue de $18,5^{\star} 100.000 \mathrm{RNV}$ $y$ en el 2012 de $18,3^{\star} 100.000 \mathrm{RNV}$.

Las causas de mortalidad materna más importante son en primer lugar: muertes obstétricas indirectas; en segundo lugar: edema, proteinuria y trastornos hipertensivos en el embarazo, parto y puerperio; en tercer lugar: complicaciones del embarazo, parto y puerperio y en último lugar el aborto.

Las tasas de mortalidad materna por estas causas desde el año 2000 a 2012 son las siguientes: la primera causa ha tenido un aumento desde 3,1 ${ }^{\star} 100.000 \mathrm{RNV}$ a $6,6{ }^{\star} 100.000 \mathrm{RNV}$, la segunda causa ha sufrido un descenso desde $4,6^{\star} 100.000$ RNV a $4,1^{\star} 100.000 \mathrm{RNV}$, la tercera causa ha disminuido desde $3,1^{\star} 100.000$ RNV a $2,5^{\star} 100.000$ RNV y el aborto como causa de mortalidad materna ha disminuido desde $5,0^{\star} 100.000 \mathrm{RNV}$ a $1,6^{\star} 100.000 \mathrm{RNV}$ (Figura 2).

Al extraer el aborto como causa de mortalidad materna, esta no sufrió ninguna alteración (no tiene peso significativo diferente a otras causas).

En la Tabla 2, se observa que el número de abortos totales van en disminución, al igual que los abortos por causas no especificadas, donde eventualmente pudiesen estar incluidos los abortos inducidos. Los abortos por esta última causa han disminuido desde $36,6 \%$ a $26,1 \%$ en el período 2001-2012 en Chile.

Durante el decenio, la mortalidad neonatal precoz, como proporción de la mortalidad infantil, se ha mantenido constante, en promedio $55,4 \%$ ( $p$-value $=0,439)$. La Figura 3 muestra la proporción de muertes de neonatos durante los

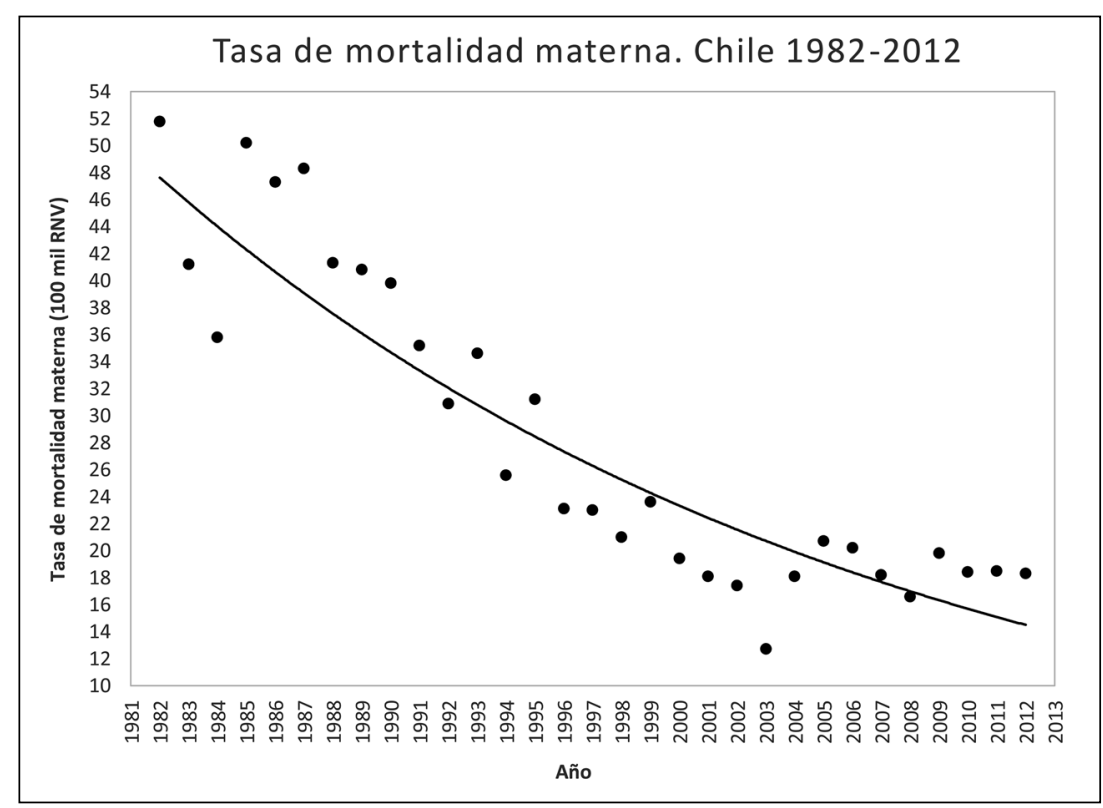

Figura 1. Tendencia de la tasa de mortalidad materna en Chile (x 100.000 RNV) desde el año 1982-2012. 


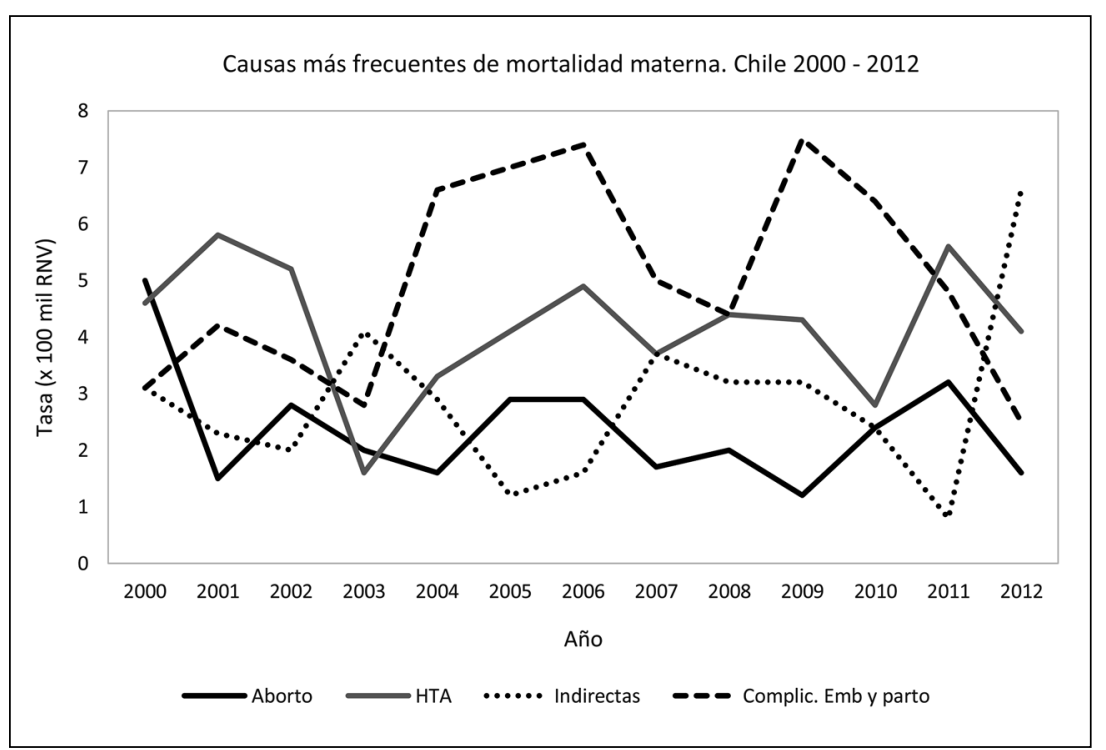

Figura 2. Tendencia de las causas más importantes de mortalidad materna en Chile (x 100.000 RNV), desde el año 2000 a 2012.

Tabla 2. Egresos por aborto en Chile 2001-2012

\begin{tabular}{|cccc|}
\hline Año & $\begin{array}{c}\text { Abortos totales } \\
\text { (CIE 10: O00-009) }\end{array}$ & $\begin{array}{c}\text { Abortos por causas } \\
\text { no especificadas (006) }\end{array}$ & $\begin{array}{c}\text { Proporción de abortos por causas no especifi- } \\
\text { cadas en relación a abortos totales (\%) }\end{array}$ \\
\hline 2001 & 33.991 & 12.436 & 36,59 \\
\hline 2002 & 34.456 & 12.971 & 37,65 \\
\hline 2003 & 33.030 & 11.970 & 36,24 \\
\hline 2004 & 33.305 & 12.113 & 36,37 \\
\hline 2005 & 32.721 & 11.646 & 35,59 \\
\hline 2006 & 32.672 & 11.213 & 34,32 \\
\hline 2007 & 32.035 & 10.980 & 34,28 \\
\hline 2008 & 32.918 & 10.914 & 33,16 \\
\hline 2009 & 33.277 & 10.247 & 30,79 \\
\hline 2010 & 31.344 & 9.785 & 31,22 \\
\hline 2011 & 30.383 & 8.751 & 28,80 \\
\hline 2012 & 30.023 & 7.838 & 26,11 \\
\hline
\end{tabular}

primeros 7 días de vida respecto de las muertes infantiles (menores de 1 año), desde el año 2000 al 2010. Posteriormente, se quiso identificar cuáles eran las principales causas de mortalidad neonatal precoz (RNV que fallecen en el puerperio, desde que nacen hasta los 7 días de vida). La Tabla 3 muestra estas causas, cuyas frecuencias acumulan sobre $50,0 \%$ de ellas.

Entre 50,0\% más frecuente se encuentran, en orden descendente: la dificultad respiratoria del recién nacido $(\mathrm{RN})$, los trastornos relacionados con la duración del embarazo, la hemorragia intracraneal no traumática, la sepsis bacteriana del RN, la hemorragia pulmonar, el síndrome de 


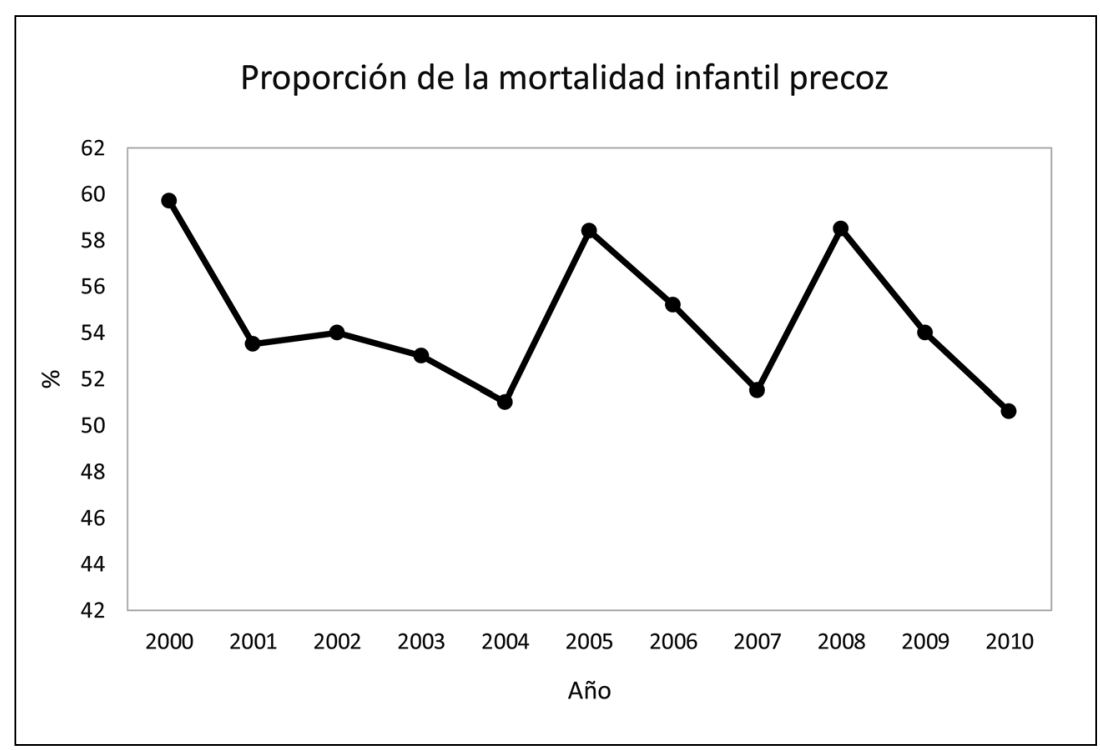

Figura 3. Proporción de la mortalidad neonatal precoz en la mortalidad infantil en Chile (\%), desde el año 2000 a 2010

Tabla 3. Principales causas de muerte neonatal precoz, Chile 2000-2010

\begin{tabular}{|lcc|}
\hline Causa & $\begin{array}{c}\text { Frecuencia } \\
\text { (n) }\end{array}$ & $\begin{array}{c}\text { Porcentaje } \\
\text { (\%) }\end{array}$ \\
\hline Dificultad respiratoria del recién nacido & 526 & 13,92 \\
\hline Trastornos relacionados con la duración corta de la gestación y con bajo peso al nacer & 418 & 11,06 \\
\hline Hemorragia intracraneal no traumática & 237 & 6,27 \\
\hline Sepsis bacteriana del recién nacido & 222 & 5,87 \\
\hline Hemorragia pulmonar originada en el período perinatal & 200 & 5,29 \\
\hline Síndrome de Edwards y síndrome de Patau & 190 & 5,03 \\
\hline Otras malformaciones congénitas del corazón & 171 & 4,53 \\
\hline
\end{tabular}

Edwards y síndrome de Patau y otras malformaciones congénitas del corazón.

De un total de 7.292 muertes en el menor de 1 año en el período 2000-2010, 2.492 ocurrieron debido a anomalías congénitas, es decir, 34,2\%. Si se analiza la mortalidad neonatal precoz en el mismo período, de 3.976 muertes, 1.310 fallecieron a causa de anomalías congénitas, es decir, 33,0\% (Figura 4).

Mediante una regresión de Poisson, se observa que la tendencia de la proporción de anomalías congénitas en muertes precoces no ha cambiado en el decenio $(p=0,763)$.

\section{Discusión}

El aborto como causa de mortalidad materna en Chile ha tenido un descenso radical entre los años 2000 y 2011, siendo esta última cifra la más baja, comparativamente, de los países de América del Sur $\left(18,3^{\star} 100.000 \mathrm{RNV}\right.$ vs $\left.44,2^{\star} 100.000 \mathrm{RNV}\right)$ e incluso una de las más bajas del continente ${ }^{10}$. En esta importante disminución ha jugado un rol fundamental la gran cobertura de control de embarazos y, especialmente, la atención profesional del parto, que alcanza $99,8 \%{ }^{11}$, la mejor de América Latina. Adicionalmente, se cuenta con importan- 


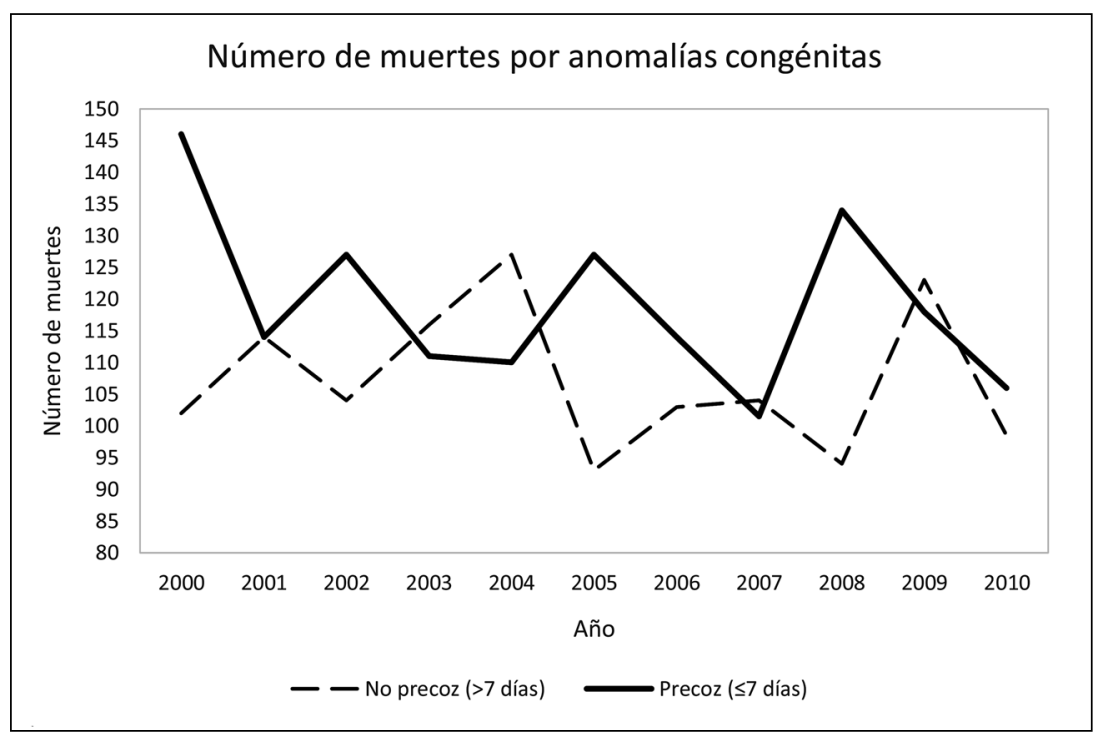

Figura 4. Número de muertes neonatales precoces y tardías por anomalías congénitas en Chile, desde el año 2000 a 2010. tes intervenciones para evitar las principales causas específicas de mortalidad materna, tales como un diagnóstico precoz y tratamiento adecuado de hipertensión arterial, diabetes mellitus o mejor manejo de complicaciones del embarazo. Otras razones fundamentales que han conducido a la importante disminución de la mortalidad materna e infantil son el sostenido desarrollo educacional, social, económico y sanitario ${ }^{12,13}$.

Entre 1990 y 2014, las tasas de aborto han disminuido significativamente en los países más desarrollados del mundo, de 46 a 27 interrupciones de embarazo anuales por cada 1.000 mujeres en edad fértil (de 15 a 44 años). Sin embargo, durante estos 24 años, en las regiones más pobres, las cifras sólo han sufrido una variación de 39 a 37 por cada 1.000 mujeres.

Los mejores resultados se registraron en el este de Europa, donde la tasa pasó de 88 abortos anuales por cada 1.000 mujeres en 1990 a 42 en 2014; en el sur de Europa, de 38 a 26 abortos anuales por cada 1.000 mujeres; en el norte de Europa de 22 a 18 abortos anuales por cada 1.000 mujeres y en América del Norte de 25 a 17 abortos anuales por cada 1.000 mujeres ${ }^{14}$.

Hay lugares en los que se ha registrado mayor proporción de abortos. Por ejemplo, en América Latina y el Caribe, la proporción aumentó de $23 \%$ a $32 \%$ en los 25 años analizados. En Asia del
Sur y Central, el porcentaje pasó de $17 \%$ a $25 \%$ y en África del Sur, de $17 \%$ a $24 \%$, desproporcionadamente afectada, ya que dos tercios de todas las defunciones relacionadas con el aborto tienen lugar en ese continente ${ }^{14}$.

Chile ha logrado disminuir en forma drástica la mortalidad materna por aborto, sin necesidad de recurrir a una legislación ${ }^{15}$ llegando a tener una tasa de $1,6^{\star} 100.000 \mathrm{RNV}$, aunque hay evidencia que sustenta el daño físico y psicológico que el aborto genera en toda mujer.

Si bien aplicamos los criterios de priorización de problemas de salud pública según la OPS-CEN$\mathrm{DES}^{12}$, el aborto como causa de muertes maternas no cumple con ellos. Estos criterios son:

a) Magnitud del problema, expresada como prevalencia o incidencia.

b) Severidad del problema, expresada como letalidad, mortalidad, años de vida saludables (AVISA) que se traducen en altos costos de atención.

c) Trascendencia del problema de salud, sería el único criterio que se cumple, pues una muerte materna o una muerte materna debida a aborto tiene implicancias clínicas y de salud pública.

d) Vulnerabilidad del problema, es decir, contar con capacidades reales para evitar el daño.

e) Evaluación de costo-efectividad de la o las intervenciones. 
En cuanto al aborto, un importante factor de riesgo es el antecedente de haber tenido un aborto previo, por tanto, en ese grupo de mujeres es necesario que precozmente se le ofrezca apoyo y consejería para prevenir futuros abortos ${ }^{16}$.

El aborto provocado es, a todas luces, un acontecimiento negativo en el plano de la salud sexual y reproductiva de toda mujer, puesto que existe certeza de los daños físicos y psicológicos que este provoca durante el ciclo de vida de la mujer.

Está claro que una consejería preventiva ante la posibilidad real de aborto es no sólo saludable, sino indispensable. Pero los contenidos de dicha consejería deben basarse en hechos objetivos y fundamentarse en el conocimiento científico existente, los que debieran enseñarse desde la etapa escolar. Solo de esta forma se podrá conducir a una medida eficiente en la prevención del aborto.

Hay estudios nacionales que demuestran que las tasas más altas de mortalidad materna se concentran en las mujeres mayores de 35 años, por ello sería recomendable definir como embarazo de alto riesgo a embarazos que ocurren en mujeres por sobre esa edad, así como mantener programas de educación continua para el personal de salud, de modo que estén capacitados para diagnosticar y tratar las enfermedades concomitantes que se puedan encontrar en mujeres de esas edades, siendo la hipertensión arterial una de las principales ${ }^{15,17}$.

Cualquier análisis que se realice en relación a mortalidad materna y sus causas debe incluir otras variables altamente relacionadas a ella, como lo son: nivel de la educación de las mujeres, programas de nutrición complementaria para mujeres embarazadas pobres, acceso a control del embarazo, acceso a centros de atención especial para la salud materna equipados con unidades de emergencia obstétricas, acceso a servicios sanitarios, los que en su conjunto pueden facilitar una transición epidemiológica en la disminución de tasas de mortalidad materna.

\section{Conclusión}

El aborto no es un problema de salud pública en Chile. Para continuar disminuyendo la mortalidad materna es necesario implementar programas de detección precoz de riesgos tales como diabetes mellitus, obesidad, hipertensión arterial, entre otras. El aborto inducido es un acontecimiento negativo en la salud sexual y reproductiva de toda mujer.

\section{Referencias}

1. United Nations Millennium Declaration. Resolution adopted by the General Assembly [Internet]. [citado el 12 de mayo de 2016]. Recuperado a partir de: http:// www.un.org/millennium/declaration/ares552e.htm

2. Organización Mundial de la Salud. OMS | Prevención del aborto peligroso [Internet]. [citado el 12 de mayo de 2016]. Recuperado a partir de: http://www.who.int/ mediacentre/factsheets/fs388/es/

3. Center of Reproductive Rights. Clasificación de las leyes sobre aborto desde la más hasta la menos restrictiva [Internet]. Recuperado a partir de: http://www. reproductiverights.org/sites/crr.civicactions.net/files/ documents/2014AbortionMapES.pdf

4. Gobierno de Chile. Proyecto de Ley que regula la despenalización de la interrupción voluntaria del embarazo en tres causales. [Internet]. Recuperado a partir de: http://www.minsegpres.gob.cl/wp-content/ upLoads/2015/01/1230-362-despenalia-interrupcion-emabrazo-3-causales-con-ingreso-camara.pdf

5. Gobierno de Chile. Objetivos Sanitarios para la década 2000-2010 [Internet]. Recuperado a partir de: http:// www.paho.org/hq/dmdocuments/2010/Politicas_Nacionales_Salud-Chile_2000-2010.pdf

6. Gobierno de Chile. Estrategia nacional de salud para el cumplimiento de los objetivos sanitarios de la década 2011-2020 [Internet]. Recuperado a partir de: http:// www.ispch.cl/sites/default/files/documento/2011/12/ Metas2011-2020.pdf

7. Ministerio de Salud. Gobierno de Chile. Departamento de estadísticas e información de salud. [Internet]. Recuperado a partir de: http://www.deis.cl/

8. Organización Mundial de la Salud (OMS), Organización Panamericana de la Salud (OPS). Clasificación Estadística Internacional de Enfermedades y Problemas Relacionados con la Salud [Internet]. Décima revisión. Vol. 1. Publicación científica $\mathrm{N}^{\circ} 554$. Washington DC; 1995. Recuperado a partir de: http://apps.who.int/classifications/apps/icd/icd10online/

9. Stata Technical Support. A publication to promote communication among Stata users. New York, 2005. [Internet]. Recuperado a partir de: http://www.stata-press. com/journals/stbcontents/stb25.pdf

10. Kassebaum NJ, Bertozzi-Villa A, Coggeshall MS, Shackelford KA, Steiner C, Heuton KR, et al. Global, regional, and national levels and causes of maternal mortality during 1990-2013: a systematic analysis for the Global Burden of Disease Study 2013. The Lancet 384 (9947): 980-1004.

11. Ministerio de Salud. Gobierno de Chile. Departamento 
de estadísticas e información de salud. Indicadores básicos de salud: Chile 2011. [Internet]. Recuperado a partir de: http://deis.minsal.cl/deis/indicadores/Folleto_IBS_2011.pdf

12. Organización Panamericana de la Salud. El método OPS-CENDES. Cap 6. [Internet]. Recuperado a partir de: http://whqlibdoc.who.int/php/WHO_PHP_46_ (part2)_spa.pdf

13. Zárate A. Un estudio de las ayudas directas a la natalidad como instrumento para incentivar la fecundidad y luchar contra la despoblación. Departamento de Estructura e Historia Económica y Economía Pública Universidad de Zaragoza. Zaragoza, 2002. [Internet]. Recuperado a partir de: http://www.ceddar.org/content/ files/articulof_272_01_DT2002-2.pdf
14. Sedgh G, Bearak J, Singh S, Bankole A, Popinchalk A, Ganatra B, et al. Abortion incidence between 1990 and 2014: global, regional, and subregional levels and trends. Lancet 2016; 388: 258-67.

15. Donoso E, Vera C. El aborto en Chile: aspectos epidemiológicos, históricos y legales. Rev Chil Obstet Ginecol 2016; 81 (6): 534-45.

16. Gissler M, Berg C, Bouvier-Colle M, Buekens P. Pregnancy-associated mortality after birth, spontaneous abortion, or induced abortion in Finland, 1987-2000. Am J Ob Gyn 2004; 190 (2): 422-7.

17. Donoso E, Carvajal J. El cambio del perfil epidemiológico de la mortalidad materna en Chile dificultará el cumplimiento del $5^{\circ}$ objetivo del Milenio. Rev Med Chile 2012; 140 (10): 1253-62. 